\title{
Die Gleichgewichtsform eines Ferrofluid-Tropfens in zweiter Näherung
}

\section{K. BEYER}

Herrn Prof. Dr. Lothar Berg zum 60. Geburtstag gewidmet

Die Arbeit enthält die Herleitung einer in zweiter Ordnung gültigen Nähèrungsformel für die Form eines Ferrofluid-Tropfens in einem homogenen Magnetfeld.

В работе содержится вывод приближениоп̆ формулы второго порядка описывающе форму капли ферромагнитной жидкости в однородном магнитном поле.

The paper contains the deduction of a second-order approximate formula for the shape of a ferrofluid drop in-a homogeneous mágnetic field.

1. In dieser Arbeit leiten wir als Ergänzung zu den Uberlégúngen in [1] eine in zweiter Ordnung gültige Näherungsformel für die Form eines Ferrofluid-Tropfens in einem homogenen Magnetfeld her. Die freie Oberfläche $S$ des (inkompressiblen) Tropfens ist durch das Prinzip der, virtuellen Arbeit

$$
\alpha S^{\prime}+E_{\mathrm{m}}^{\prime}-\lambda \Omega^{\prime}=0
$$

bestimmt, wobei $\alpha>0$ die Oberflächenspannung, $S$ die Oberfläche und $\Omega$ dás Volumen des Tropfens sowie $E_{\mathrm{m}}$ die freie magnetische Eńergie bezeichnen. Bei linearem Zusammenhang zwischen Feldstärke und Magnetisierung $\vec{M}$ ist $E_{\mathrm{m}}$ gemä $\vec{B}$

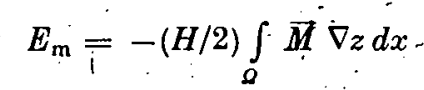

zu berechnen [2]; $H \nabla z$ beschreibt das freie Parallelfeld. Die Einführung des Lagrangeschen Multiplikators $\lambda$ in (i) trägt der Volumenkonstanz der tatsächlichen Vergleichskonfigurationen Rechnung. Im folgenden schreiben wir die Volumina mit $\Omega=4 \pi / 3$ vor. Wie in (1) und (2) werden $S$-und $\Omega$ in diesem Text sowohl zur Bezeich: nung einer Menge aly auch ihres Maßes benutzt.

Nach. Satz 1 [1] lassen sich die (bis auf Translationen) eindeutig bestimmten Lösungen von (1) nach dem voraussetzungsgemäß kleinen Quotienten $\varepsilon=B^{2} / 4 \pi \alpha$ analytisch entwickeln. Genauer : Parametrisiert man die zur Konkurrenz gelangenden Flächen durch

$$
\bar{S}=\left\{(1+u(x)) x: x=(x, y, z) \in S^{2}\right\},
$$

dann gilt $u=\sum \varepsilon^{n} u_{n}$. Unter der Voraussetzung.s $>2$ konvergiert die Reihe bezüg- lich der Sobolev-Metrik $\left\|u ; H^{s}\left(S^{2}\right)\right\|=\left(\left\|\Lambda^{s} u ; L^{2}\left(S^{2}\right)\right\|^{2}+\left\|u ; L^{2}\left(S^{2}\right)\right\|^{2}\right)^{1 / 2}$. In dieser Vorschrift bedeutet $\Lambda=(-\Delta)^{1 / 2}$ und

$$
\Delta=\frac{1}{\sin \vartheta} \frac{\partial}{\partial \vartheta} \sin \vartheta \frac{\partial}{\partial \vartheta}+\frac{1}{\sin ^{2} \vartheta} \frac{\partial^{2}}{\partial \varphi^{2}}
$$


den Laplace-Operator der Sphäre $S^{2}$, bezogen auf die Kugelkoordinaten

$$
x=r \sin \vartheta \cos \varphi, \quad y=r \sin \vartheta \sin \varphi ; \quad z=r \cos \vartheta
$$

Die Lösungsflächen besitzen neben einer horizontalen Symmetrieebene Rotationssymmetrie bezüglich der $z$-Achse.

Wir berechnen hier neben dem schon in [1] angegebenen $u_{1}$ (dort mit einem fehlerhaften Faktor) auch den zweiten Koeffizienten $u_{2}$ der obigen Reihenentwicklung.

2. Wir beginnen mit den Entwicklungen für Oberfläche

$$
\begin{aligned}
& S=S(u)=\int_{S^{2}}(1+u)^{2}\left(1+|\nabla u|^{2} /(1+u)^{2}\right)^{1 / 2} d S^{2} \\
& \because=4 \pi+\int_{S^{2}}\left(2 u+u^{2}+2^{-1}|\nabla u|^{2}\right) d S^{2}+\grave{O}\left(u^{4}\right)
\end{aligned}
$$

(der Gradient bezieht sich hier sowie später an entsprechender Stelle auf die Sphäre $\left.S^{2}\right)$ und Volumen $\Omega=\int_{S^{2}}(1+u)^{3} d S^{2} / 3$ der Figur (3).

Die màgnetische Energie (2) läßt sich durch den Minimalwert $E$ des (bei fixiertem u) quadratischen Variationsproblems

$$
2^{-1} \int_{\mathbb{R}^{2}} \mu|\nabla \psi|^{2} d x+(\mu-1) \int_{\Omega} \partial \psi / \partial z d x \rightarrow \dot{M i n}
$$

wie folgt ausdrücken (s. [1]):

$$
E_{\mathrm{m}}=-H^{2}\left(\chi^{\Omega / 2}+E / 4 \pi\right) \quad \text { mit } E=-2^{-i} \int_{\mathbb{R}^{2}} \mu|\nabla \psi|^{2} d x
$$

$\chi \doteq(\mu-1) / 4 \pi$ ist die nach Voraussetzung konstante magnetische Suszeptibilität des Ferrofluids. Vereinbarungsgemä $B$ ist bei der Integration über das (nichtmagnetische) Komplementärgebiet $C \bar{\Omega}$ stets $\mu$ gleich eins zu setzen. Zur Entwicklung von $E: E=E(0)+\left\langle E^{\prime}(0), u\right\rangle+2^{-1} E^{\prime \prime}(0)\left\{u^{2}\right\}+O\left(u^{3}\right)$ ist neben der Konfiguration $u$ - (bzw. $S=S(u)$ ) eine zu $u+t v$ gehörige Schar $S_{t}=S(u+t v)$ von Nachbarflächen einzuführen. Das nach (4) zu konstruierende Magnetfeld zu $S_{t}$ sei $\psi(t, x)$. Seine partiellen. Ableitungen $\partial \psi / \partial t$ nach der Variablen $t$ bezeichnen wir abkiirzend mit $\dot{\psi}$ : $\dot{\psi}(x)=\partial \psi(0, x) / \partial t$. Wie die Felder $\psi$ hängen die Funktionen $\dot{\psi}$ von der Konfiguration $u \mathrm{ab}$, dazu tritt zusätzlich die (lineare) Abhängigkeit von ihrer Variàtion $v$. Im Text sind diese Variablen dem jeweiligen Zusammenhang zu entnehmen. Mit oberen Signaturen kennzeichnen wir schließlich die Einschränkungen $\psi^{-}=\psi_{1}, \psi^{+}=\psi_{1 \mathrm{C} \Omega}$ auf $\Omega$ bzw. $C \bar{\Omega}$ und ihre Grenzwerte längs $S$.

Die erste Variation des Minimalwertes $E$ ist durch Differentiation des Integrals $E(u+t v)$ zu bestimmen:

$$
\left\langle E^{\prime}(u), v\right\rangle=-\int_{\mathbb{R}^{2}} \mu \nabla \psi \nabla \dot{\psi} d x-2^{-1} \int_{S}\left(\mu\left|\nabla \psi^{-}\right|^{2}-\left|\nabla \psi^{+}\right|^{2}\right)(1+u)^{2} v d S^{2} .
$$

Āhnlich folgt aus den Variationsgleichungen

$$
\int_{\mathbb{R}^{\prime}} \mu \nabla \psi \nabla \varphi d x+(\mu-1) \int_{\Omega} \partial \varphi / \partial z d x=0
$$

zu (4) durch Differentiation

$$
\int_{\mathbb{R}^{2}} \mu \nabla \dot{\psi} \nabla \varphi d x+\int_{S}\left(\mu \cdot \nabla \psi^{-}-\nabla \psi^{+}+(\mu-1) \nabla z\right) \nabla \varphi(I+u)^{2} v d S^{2}=0
$$


$\mathrm{Zu}$ (7) gehören die Eulerschen Gleichungen

$$
\begin{aligned}
& \Delta \psi=0 \quad \text { in } \quad \mathbb{R}^{3} \backslash S, \\
& \psi^{-}-\psi^{+}=0, \quad \mu \partial \psi^{-} / \partial n-\partial \psi^{+} / \partial n+(\mu-1) \partial z / \partial n=0 \text { längs } S,
\end{aligned}
$$

aus denen im Spezialfall $u^{\prime}=0$

$$
\psi^{-}=A r \cos \vartheta, \quad \psi^{+}=\left(A / r^{2}\right) \cos \vartheta \quad \text { mit } A=(1-\bar{\mu}) /(2+\mu)
$$

folgt. Allgemein gilt wegen der Sprungrelationen: In (8) ist die Linearform

$$
\varphi \rightarrow \int_{S} \nabla\left(\mu \psi^{-}-\psi^{+}+(\mu-1) z\right) \nabla \varphi(1+u)^{2} v d S^{2}
$$

nur von den Randwerten $\varphi_{\mid S}$ abhängig. Das impliziert

$$
\begin{aligned}
\int_{\mathbb{R}^{3}} \mu \nabla \dot{\psi} \nabla \psi d x= & -2^{-1} \int_{S} \nabla\left(\mu \psi^{-}=\psi^{+}+(\mu-1) z\right) \\
& \times \nabla\left(\psi^{-}+\psi^{+}\right)(1+u)^{2} v d S^{2},
\end{aligned}
$$

wonach (6) in der Form

$$
\left\langle E^{\prime}(u), v\right\rangle=2^{-1}(\mu-1) \int_{S}\left(\nabla \psi^{-} \nabla \psi^{+}+\psi_{z}^{-}+\psi_{z}^{+}\right)(1+u)^{2} v d S^{2}
$$

geschrieben werden kann.

Erneute Differentiation führt zur zweiten Variation, deren Werte wir hịer nur für $u=0$ notieren :

$$
\begin{aligned}
E^{\prime \prime}(0)\{v, w\}= & 2^{-1}(\mu-1) \int_{S^{z}}\left(\nabla \dot{\psi}^{-} \nabla \psi^{+}+\nabla \psi^{-} \nabla \dot{\psi}^{+}+\dot{\psi}_{z}^{-}+\dot{\psi}_{z}^{+}\right. \\
& +w \partial / \partial r\left(\nabla \psi^{-} \nabla \psi^{+}+\psi_{z}^{-}+\psi_{z}^{+}\right) \\
& \left.+2 w\left(\nabla \psi^{-} \nabla \psi^{+}+\psi_{z}^{-}+\psi_{z}^{+}\right)\right) v d S^{2}
\end{aligned}
$$

In dieser Formel ist $\dot{\psi}$ zur Variation $w$ zu bilden; im Hinblick auf (8), (9) ist dazu das Kopplungsproblem

$$
\begin{aligned}
& \Delta \psi=0 \quad \text { in } \mathbb{R}^{3} \backslash S^{2} \\
& \mu \partial \dot{\psi}^{-} / \partial n-\partial \dot{\psi}^{+} / \partial n=\operatorname{div}\left(w \nabla\left(\mu \psi^{-}-\psi^{+}+(\mu-1) z\right)\right) \\
& \dot{\psi}^{-}-\dot{\psi}^{+}=u\left(\partial \psi^{+} / \partial n-\partial \psi^{-} / \partial n\right) \quad \text { längs } S^{2}
\end{aligned}
$$

zu lösen (dir und $\nabla$ beziehen sich auf die Sphäre $S^{2}$ ).

Die Nebenbedingung $\Omega(u)=4 \pi / 3$ ist (lokal) äquivalent zu

$$
u=v+f(v) 1_{\mid S^{2}}, \quad v \in 1_{\mid S^{3}}^{1}
$$

$\operatorname{mit}_{\text {ein }} f(v) \doteq-\int_{s^{1}}\left(v^{2}+v^{3} / 3\right) d S^{2} / 4 \pi+O\left(v^{4}\right)$. Führen wir (14) in unsere Entwicklungen

$$
\begin{aligned}
\tilde{S}(v)= & S(v+f(v) 1)=4 \pi+\int_{S^{2}}\left(2^{-1}|\nabla v|^{2}-v^{2}-(2 / 3) v^{3}\right) d S^{2}+O\left(v^{4}\right) \\
\tilde{E}(v)= & E(v+f(v) 1) \\
= & E(0)+\left\langle E^{\prime}(0), v\right\rangle \\
& -(4 \pi)^{-1}\left\langle E^{\prime}(0) ; 1\right\rangle \int_{S^{2}} v^{2} d S^{2}+2^{-1} E^{\prime \prime}(0)\left\{v^{2}\right\}+O\left(v^{3}\right)
\end{aligned}
$$


dann geht die Gleichgewichtsbedingung (1) in

$$
\tilde{S}^{\prime}(v)-\varepsilon \tilde{E}^{\prime}(v)=0, \quad \varepsilon=H^{2} / 4 \pi \alpha,
$$

über; man beachte (5).

Bemerkung: (15) kann durch die Gleichungen

$$
\left\langle\tilde{S}^{\prime}(v)-\varepsilon E^{\prime}(v), w\right\rangle_{-}=0 \quad \text { für alle } w \in 1^{1} \cap L^{1}
$$

ersetzt werden, wobei $L$ die lineare Hülle der Funktionen $\left.x_{i_{\mid} S^{1}} \overline{(i}=1,2,3\right)$ bereichnet.

Beweis: Mit der Abkürzung $F=S-\varepsilon E$ folgt aus (15)

$$
\begin{aligned}
0 & =\left\langle F^{\prime}(u), w+f^{\prime}(v)\{w\} 1\right\rangle=\left\langle F^{\prime}(u), w\right\rangle+f^{\prime}(v)\{w\}\left\langle F^{\prime}(u), 1\right\rangle \\
& =\left\langle F^{\prime}(u), w\right\rangle-\lambda\left\langle\Omega^{\prime}(u), w\right\rangle \quad \text { für } w \in L^{\perp}
\end{aligned}
$$

und $\lambda=F^{\prime}(u) 1 / \overrightarrow{\Omega^{\prime}}(u) 1$. Die restlichen Gleichgewichts bedingungen

$$
\left\langle F^{\prime}(u)-\varepsilon \Omega^{\prime}(u), x_{i \mid S^{2}}\right\rangle=0 \quad-(i=1,2,3)
$$

sind wegen der Translationsinvarianz der beteiligten Funktionale automatisch erfüllt. In der Tat gilt für translationsinvariantes $J$

$$
\left\langle J^{\prime}(u) ; x_{i}-\nabla x_{i} \nabla u /(1+u)\right\rangle=0 \quad(i=1,2,3)
$$

identisch in $u$, woraus $\left\langle J^{\prime}(u), x_{i}\right\rangle=0$ folgt, sofern $\left\langle J^{\prime}(u), w\right\rangle=0$ für $w \in L^{\perp}$ zutrifft

Nach [1] besitzt (16) eine eindeutig bestimmte Lösung $v \in 1^{\perp} \cap L^{\perp}$ mit der Entwicklung $v=\varepsilon v_{1}+\varepsilon^{2} v_{2}+O\left(\varepsilon^{3}\right), v_{i} \in 1^{\perp} \cap L^{\perp}$. Einsètzen und Koeffizientenvergleich führt zu.

$$
\begin{aligned}
\int_{S^{2}}\left(\nabla v_{1} \nabla w-2 v_{1} w\right) d S^{2} & =\left\langle E^{\prime}(0), w\right\rangle \\
\int_{S^{2}}\left(\nabla v_{2} \nabla w-2 v_{2} w\right) d S^{2} & =g(w) \\
& =\int_{S^{2}}\left(2 v_{1}^{2}-(2 \pi)^{-1} v_{1} E^{\prime}(0) 1\right) w d S^{2}+E^{\prime \prime}(0)\left\{v_{1}, \grave{w}\right\}
\end{aligned}
$$

Da nach (10), (11)

$$
\left\langle E^{\prime}(0), v\right\rangle=2^{-1} A^{2} \int_{S^{2}}\left(6 P_{2}(\cos \vartheta):-(\mu+2)\right)^{\prime} v d S^{2}
$$

gilt ( $P_{i}$ sind die Legendreschen Polynome), bedeutet (17), daß

$$
-\Delta v_{1}-2 v_{1}=3 A^{2} P_{2}(\cos \vartheta), \quad \text { d. h. } \quad v_{1} \doteq(3 / 4) A^{2} P_{2}(\cos \vartheta)
$$

ist. $v_{2}$ ist in zwei Schritten zu berechnen:

1. Lösung des Kopplungsproblems (13)

$$
\begin{aligned}
& \Delta \dot{\psi}=0, \quad \text { in } \mathbb{R}^{3} \backslash S^{2}, \\
& \mu \frac{\partial \dot{\psi}^{-}}{\partial n}-\frac{\partial \dot{\psi}^{+}}{\partial n}=3 A \frac{1}{\sin \vartheta} \frac{\partial}{\partial \vartheta}\left(\vartheta_{1} \sin ^{2} \vartheta\right)=\frac{9}{20} A^{3}\left(-2 P_{1}+12 P_{3}\right) \\
& \dot{\psi}^{-}-\dot{\psi}^{+}=-3 v_{1} \cos \vartheta=-(9 / 20) A^{3}\left(2 P_{1}+3 P_{3}\right) .
\end{aligned}
$$


Die Lösung lautet

$$
\dot{\psi}^{-}=-\frac{27}{10} \frac{A^{3}}{\mu+2} r P_{1}, \quad \dot{\psi}^{+}=-\frac{9}{10} A^{3}\left(\frac{A}{r^{2}} P_{-1 .}-\frac{3}{2} \frac{P_{3}}{r^{4}}\right) .
$$

2. Einsetzen in (12) bzw. (18):

$$
\begin{aligned}
E^{\prime \prime}(0)\left\{v_{1}, w\right\} & =E^{\prime \prime}(0)\left\{w, v_{1}\right\}^{\prime} \\
= & 2^{-1}(\mu-1) \int_{S^{3}}\left(\dot{\psi}_{z}^{-} \psi_{z}^{+}+\psi_{z} \dot{\psi}_{z}^{+}+\dot{\psi}_{z}^{-}+\dot{\psi}_{z}^{+}\right. \\
& \left.\quad+v_{1}(\partial / \partial z+2)\left(\psi_{z}{ }^{-} \psi_{z}^{+}+\psi_{z}^{-}+\psi_{z}^{+}\right)\right) w d S^{2} \\
= & 3 A^{4} \int_{S^{3}}\left(\frac{27}{10} P_{4}-\frac{3}{4} P_{2}{ }^{2}-\left(\frac{2+\mu}{4}+\frac{9}{5} A\right) P_{2}\right) w d S^{2},
\end{aligned}
$$

(für $\left.w \in 1^{1}\right) \mathrm{bzw}$.

$$
g(w)=9 A^{4} \int_{S^{2}}\left(\frac{117}{140} P_{4}-\left(\frac{1}{28}+\frac{3}{5} A\right) P_{2}\right) w d S^{2}
$$

Von (18) lesen wir jetzt

$$
v_{2}=\frac{9}{4} A^{4}\left(\frac{13}{70} P_{4}-\left(\frac{1}{28}+\frac{3}{5} A\right) P_{2}\right)
$$

ab. Faßt man die Entwicklungen für $v$ und $u$ zúsammen, so folgt endgültig.

$$
\begin{aligned}
& u=\frac{3}{4} A^{2} \varepsilon P_{2}(\cos \vartheta) \\
& \quad+\frac{9}{4} A^{4} \varepsilon^{2}\left(-\frac{1}{20}-\left(\frac{1}{28}+\frac{3}{5} A\right) P_{2}(\cos \vartheta)+\frac{13}{70} P_{4}(\cos \vartheta)\right)+\dot{O}\left(\varepsilon^{3}\right)
\end{aligned}
$$

\section{LITERATUR}

[1] ВеуеR, K.: Gleichgewichtsfiguren mَagnetischèr Flüssigkeiten. Z. Anal. Anw. 7.(1988), $67-76$.

[2] Landau, L. D., und E. M. Lifsciitz: Lehrbuch der theoretischen Physik, Bd. VIII. Berlin: Akademie-Verlag 1974.

Manuskripteingang: 21.04. 1989

VERFASSER :

Prof. Dr. Klads Beyer

Fachbereich Mathematik der Universität

Augustusplatz 6

D : 0 - 7010 Leipzig 